International Journal of Biomedicine I June 2019 - Volume 9, Issue Suppl_1: Abstracts From the Second Russian International Conference "Cryo-electron microscopy 2019: achievements and prospects"

POSTER ABSTRACT PRESENTATIONS

SESSION TITLE: STRUCTURE OF MEMBRANE PROTEINS

DOI: 10.21103/IJBM.9.Suppl_1.P9

\title{
Abstract P-9: Immobilization of Membrane Proteins for Single-Molecule Microscopy Studies
}

Aizada Nurdinova, Ivan Maslov, Polina Khorn, Nadezhda Safronova, Nickolay Ilyinsky, Pavel Kuzmichev, Valentin Borshchevskiy Moscow Institute of Physics and Technology, Dolgoprudny, Russia

Background: Single-molecule fluorescence microscopy studies can reveal conformational changes in proteins, help to find their stable states and to investigate transitions kinetics. The major advantage of this method is the possibility to show the heterogeneity of the properties within the ensemble. Single molecules can be observed in two different ways: in solution, where they can freely diffuse, and immobilized on the surface. The latter allows to significantly increase the observation time of the molecule.

Methods: Single-molecule fluorescence measurements were performed using microscope LSM780, Carl Zeiss at MIPT. Quartz slides were cleaned, passivated with polyethylene glycol (PEG), and coated with streptavidin. Biotin conjugated samples were introduced into the sample chamber and allowed to bind to the streptavidin-coated surface, after which unbound molecules were washed away with PBS. The two membrane systems were sampled in this work: nanodiscs and liposomes.

Results: In this work, the immobilization protocol have been developed and thoroughly tested. Each layer of the immobilization system has been checked sequentially on specificity. It turned out that streptavidin attaches to the surface covered with 3\% biotin-PEG and has free binding sites for biotinconjugated molecules. Nonspecificity observed when the nanodiscs containing $5 \%$ of biotinylated lipids are added to the system. Moreover, the usage of longer biotinylated lipids in nanodiscs does not help. Tests made with liposomes containing $5 \%$ of biotinylated lipids showed specific binding. Single-molecule measurements are possible at a concentration about $10 \mathrm{pMol}$.

Conclusion: Attempts to catch a membrane protein were made in this experiment. Unfortunately, nanodiscs immobilization did not show the desired specificity. However, the liposomes look very promising in this context.

Key Words: single-molecule microscopy $\bullet$ immobilization $\bullet$ membrane systems 
Sources of Funding: The work was performed in the framework of governmental task of Ministry of Education and Science of the Russian Federation, project № 6.9909.2017/6.7

International Journal of Biomedicine. 2019;9 Suppl 1: S20. doi: 10.21103/IJBM.9.Suppl_1.P9 (C)2019 International Medical Research and Development Corporation 